\title{
COUNTERROTATING STARS IN THE DISK OF THE Sab GALAXY NGC 7217
}

\author{
MiCHAEL R. MERRIFIELD \\ Department of Physics, University of Southampton, Highfield SO9 5NH, UK \\ mm@sousun1.phys.soton.ac.uk \\ AND \\ KONRAD KUIJKEN \\ Harvard-Smithsonian Center for Astrophysics, 60 Garden Street, Cambridge, MA 02138 \\ kuijken@cfa.harvard.edu \\ Received 1993 May 25; accepted 1994 March 15
}

\begin{abstract}
We have analyzed high signal-to-noise spectra of the disk galaxy NGC 7217 in order to extract the full line-of-sight velocity distribution along both its major and minor axes. The data reveal that $20 \%-30 \%$ of the stars in this galaxy are in a distinct component on retrograde orbits. This counterrotating population cannot be explained away as a systematic error, and it does not seem to be caused by the bulge's contribution to the velocity distribution. We have developed a new technique for fitting dynamical disk models directly to the galaxy spectra, and application of this method confirms the presence of the distinct counterrotating disk population.

NGC 7217 is only the second disk galaxy known to contain counterrotating stars, but we argue that similar components in other regular disk systems would not have been detected by traditional techniques, and so there could exist many such systems. The existence of disk stars on retrograde orbits provides a new clue as to the manner in which the galaxy formed: it favors a scenario in which matter continues to accrete onto the galaxy over a long period of time, with rapid, substantial changes occurring in the angular momentum of the infalling material. The observable consequences of this evolutionary history include a large bulge-to-disk ratio and the absence of strong spiral structure, and so the presence or absence of a counterrotating component may go some way toward explaining the Hubble sequence for disk galaxies.
\end{abstract}

Subject headings: galaxies: individual (NGC 7217) — galaxies: kinematics and dynamics — galaxies: spiral

\section{INTRODUCTION}

The present-day distribution of stars in galaxies can provide us with important clues which help further our understanding of the processes of galaxy formation and evolution. Gross variations in overall morphology, such as the distinction between elliptical and disk systems, clearly point to very different life histories, but a great deal of information can also be gleaned from subtler variations in the kinematic properties of stars in galaxies. For example, the distribution of stellar velocities perpendicular to the plane of a disk galaxy constrain theories of the dynamical processes which heat the disk stars (reviewed by Lacey 1991), and the discovery of "kinematically distinct" nuclei in elliptical galaxies has been used to argue that these systems cannot have formed in a single epoch of collapse (Kormendy 1984). Stellar-dynamical measurements of the gravitational potential in galaxies also benefit greatly from use of the full velocity distribution (Kuijken 1991; Merritt \& Saha 1993), and in face-on galaxies the line-of-sight kinematics can be used to reconstruct the complete vertical density distribution of the disk (Merrifield 1991). It therefore behooves us to study the detailed kinematics of stars in galaxies as well as their spatial distribution.

In practice, only the velocity component down the line of sight is accessible to observations. Since the absorption-line spectrum of a galaxy is the convolution of a mean stellar spectrum with the line-of-sight velocity distribution (LOSVD) of the stars, it is possible, at least in principle, to deconvolve the LOSVD from spectral observations of the galaxy if we have a suitable stellar template spectrum. Although this analysis only provides us with the distribution of one of the three velocity components, knowledge of the LOSVD still offers a whole extra dimension of information. Indeed, since the stellar kinematics in most realistic galaxy potentials are governed by just three integrals of motion (Binney \& Tremaine 1987), a threedimensional data set (such as the LOSVD at every point on the face of a galaxy) should go a long way toward determining the entire phase-space distribution of the stars in the system.

To date, most studies of the line-of-sight kinematics in galaxies have concentrated on extracting a mean speed and a velocity dispersion, assuming that the LOSVD has a Gaussian shape (mainly based on the algorithms devised by Tonry \& Davis 1979 and Sargent et al. 1977). Although these parameters are useful in defining the gross properties of the velocity distribution, the systematic effects that result when the true LOSVD is not Gaussian are poorly understood. Quantitative analysis of these parameters, based on their substitution into the Jeans equations (which relate the moments of the velocity distribution), can be seriously compromised by such systematic errors. In order to correct for these errors, we need to know the true shape of the LOSVD.

A number of different methods for studying the full LOSVD have recently been developed (Franx \& Illingworth 1988; Bender 1990; Rix \& White 1990, 1992; van der Marel \& Franx 1993; Kuijken \& Merrifield 1993; Saha \& Williams 1993); for a discussion of these methods, see Kuijken \& Merrifield (1993, henceforth Paper I). Most of the applications of these methods have so far been focused on elliptical galaxies, but the same ideas are applicable to disk galaxies, where the shape of the LOSVD is likely to depart significantly from a Gaussian. In our own Milky Way, for example, the local distribution of 
TABLE 1

Basic Properties of NGC 7217

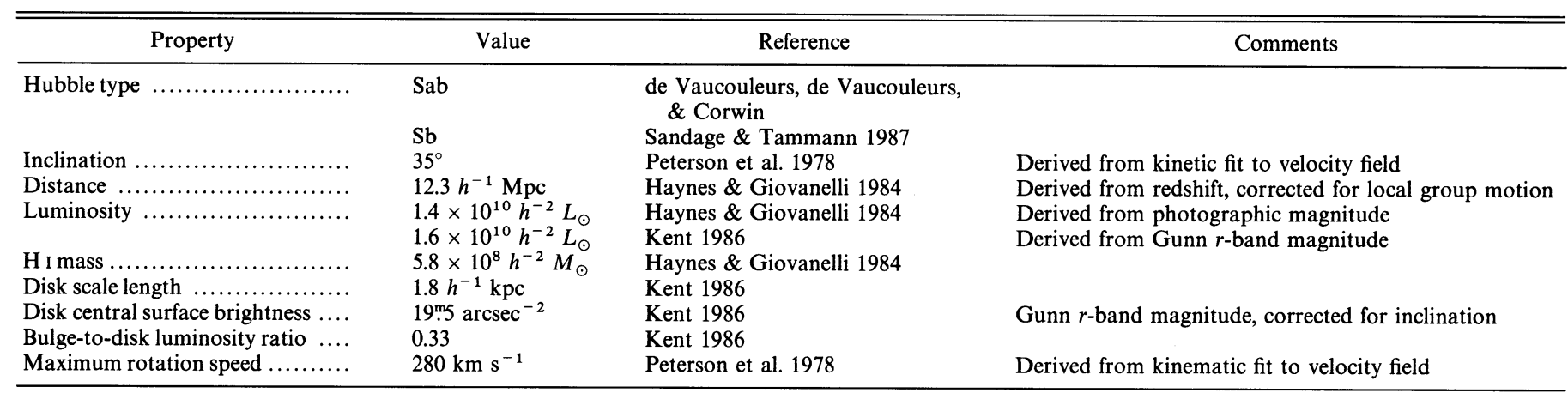

NoTE.-Quantities scaled for a Hubble constant of $H_{0}=100 \mathrm{hm} \mathrm{s}^{-1} \mathrm{Mpc}^{-1}$.

azimuthal stellar motions shows a sizeable asymmetric tail toward slower rotation velocities (Erickson 1975). In order to examine such features in the kinematics of other galaxies, we have developed a new technique-unresolved Gaussian decomposition (UGD)-for deconvolving the full LOSVD from galaxy absorption-line spectra (Paper I). This method models the LOSVD as a sum of overlapping Gaussian velocity distributions; by varying the amplitude of these Gaussians, we can reproduce any smoothly varying velocity distribution.

As a first detailed application of the method we chose the nearby regular Sab galaxy, NGC 7217, for study. The observed properties of this system are summarized in Table 1 , and a CCD image is presented in Figure 1 (Plate 8). Since this image shows no asymmetric distortions such as a bar, strong dust lanes, or strong spiral structure, the starlight that we see should come from objects on regular orbits in an axisymmetric potential. The only slight photometric peculiarity in this galaxy is that it contains a ring: as can be seen from the major-axis luminosity profile presented in Figure 2, the surface brightness at a radius of $\sim 90^{\prime \prime}$ rises almost a magnitude above the extrapolation of an exponential fitted to the inner part of the

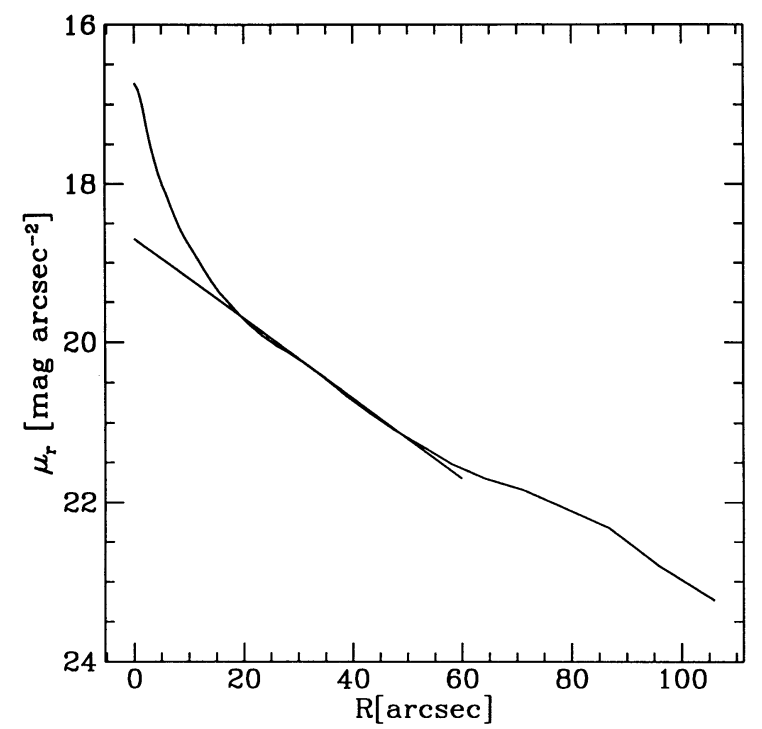

FIG. 2.-Major-axis luminosity profile of NGC 7217. The line shows an exponential fit to the disk luminosity in the inner two scale lengths ( $r$-band photometry from Kent 1986). disk. However, this feature does not point toward any significant deviation from axisymmetry, and so the stellar dynamics of the galaxy should still be reasonably tractable.

Our original intention had been to take advantage of the intermediate inclination of the disk in this system in order to extract all three components of the velocity dispersion, $\sigma_{R}, \sigma_{\phi}$, and $\sigma_{z}$ in cylindrical polar coordinates $(R, \phi, z)$. We planned to achieve this goal by observing the line-of-sight velocity dispersions along both the major and the minor axes of the system, allowing for any departures from a Gaussian distribution revealed by the UGD algorithm. By simple geometry, these observable moments of the line-of-sight velocity distribution can be written $\left(\sigma_{\phi}^{2} \sin ^{2} i+\sigma_{z}^{2} \cos ^{2} i\right)$ and $\left(\sigma_{R}^{2} \sin ^{2} i+\sigma_{z}^{2} \cos ^{2} i\right)$, respectively. The variations in these quantities with radius are supplemented by the relation between $\sigma_{R}$ and $\sigma_{\phi}$ derived from the epicycle approximation,

$$
\frac{\sigma_{\phi}^{2}}{\sigma_{R}^{2}}=\frac{1}{2 v_{c}} \frac{\partial\left(R v_{c}\right)}{\partial R},
$$

where $v_{c}(R)$ is the circular speed as derived from an emissionline rotation curve. The combination of these three constraints should hence have allowed us to solve for the size of all three components of the stellar velocity dispersion as a function of radius in the disk of the galaxy.

As it turned out, NGC 7217 proved to be totally unsuitable for this analysis. The epicycle approximation, which assumes that the actual orbital structure is a small perturbation from a disk in purely circular motion, spectacularly fails to apply in this galaxy: our analysis of the LOSVDs revealed that a significant fraction of the disk stars (20\%-30\%) circulate NGC 7217 in the opposite sense to the majority. A similar counterrotating stellar population has been found in one other disk galaxy, NGC 4550 (Rubin, Graham, \& Kenney 1992; Rix et al. 1992). In the case of NGC 4550, approximately equal numbers of the stars rotate in each direction, which made the counterrotating nature of the system apparent even in the raw spectra; what is particularly interesting about NGC 7217 is that the counterrotating component was only revealed after a detailed analysis of the LOSVD - an analysis to which few disk galaxies have been subjected. It is therefore quite possible that similar counterrotating populations lie undetected in many disk systems. Such a class of objects appears closely related to the counterrotating gas disks discovered in a significant fraction of early-type disk galaxies (Galletta 1987; Bettoni, Fasano, \& Galletta 1990; Bettoni, Galletta, \& Oosterloo 1991; Bertola, 
PLATE 8

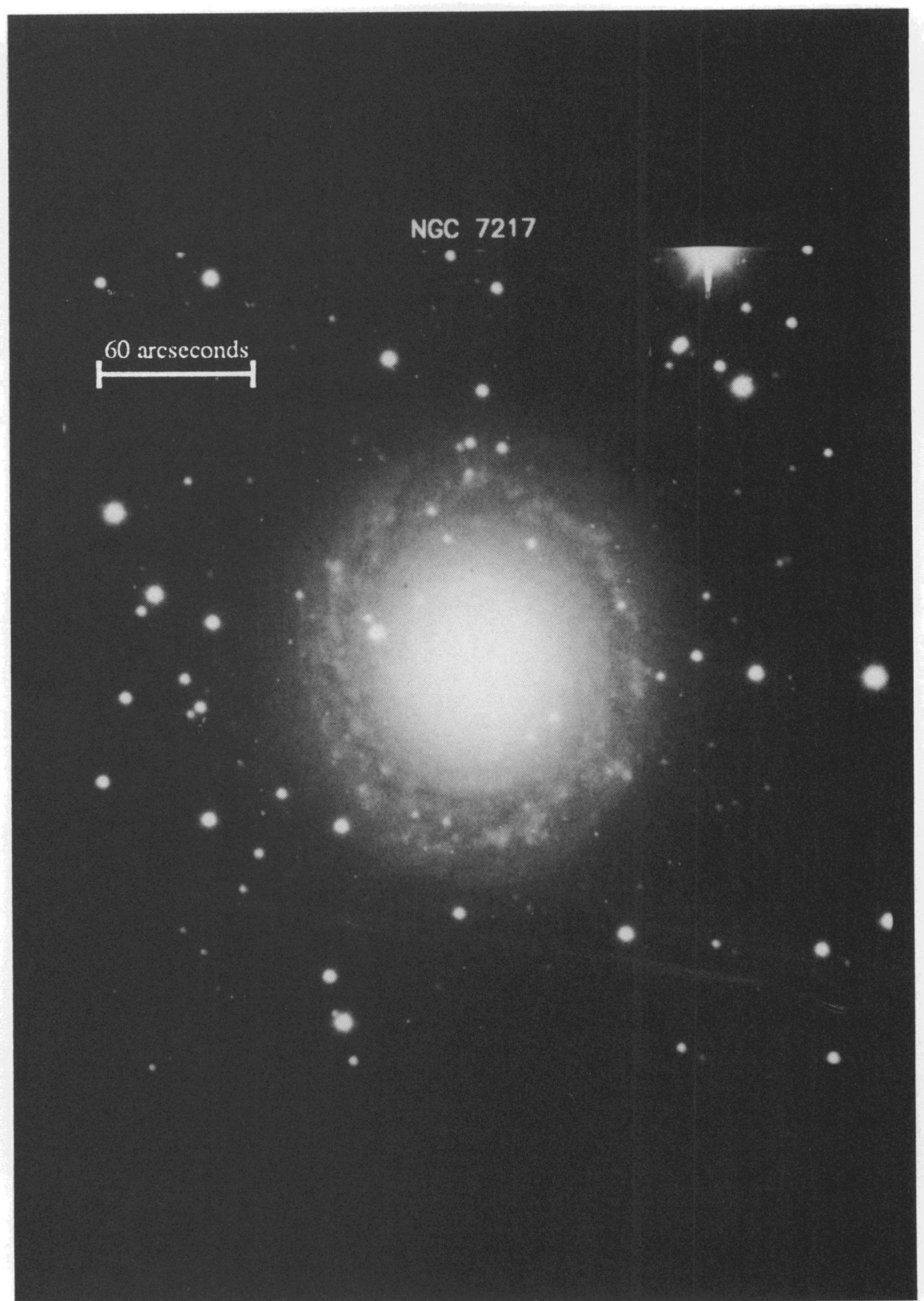

FIG. 1.-Combined $B-, V$-, and $I$-band CCD image of NGC 7217 obtained with the 48 inch telescope of the Whipple Observatory, Mount Hopkins. East is at the top, north at the left of the image.

MERRIFIELD \& KUIJKEN (see 432, 576) 
Buson, \& Zeiliger 1992) and may also be connected with other kinematically misaligned phenomena, such as polar ring galaxies (Casertano, Sackett, \& Briggs 1991) and the counterrotating stellar (Bender 1988; Franx \& Illingworth 1988; Jedrzejewski \& Schechter 1989) and gaseous (Bertola \& Bettoni 1988) cores found in many elliptical galaxies. Study of the detailed dynamics of such systems promises to provide important clues as to their formation and current state of evolution.

The remainder of this paper presents the results of our kinematic analysis of NGC 7217. The next section (§ 2) describes the spectral observations and the LOSVDs that were derived from this data set. In $\S 3$, we present a number of tests which demonstrate that the counterrotating component that we find in these velocity distributions cannot result from noise in the observations or systematic effects due to template mismatch. Section 4 turns to the dynamical implications of the observations and shows that the counterrotation in NGC 7217 must almost certainly be attributed to stars on retrograde orbits in its disk. In order to obtain a more quantitative description of the full phase-space distribution of stars in the galaxy's disk, we have also fitted truly dynamical models (i.e., distribution functions which satisfy the collisionless Boltzmann equation) directly to the spectra - the properties of these disk models are described in $\S 5$, and the details of the fitting procedure and the resulting best-fit distribution functions are presented in $\S 6$. The dynamical modeling also consistently returns distribution functions with a significant retrograde component, and in $\S 7$ we discuss these results, their impact on other observable properties of NGC 7217, and their possible implications for theories of galaxy formation.

\section{THE LINE-OF-SIGHT VELOCITY DISTRIBUTION IN NGC 7217}

We obtained long $(4.5 \mathrm{hr})$ integrations along the major (at a position angle of $86^{\circ}$ ) and minor (at a position angle of $176^{\circ}$ ) axes of the Sab galaxy NGC 7217 with the Canada-FranceHawaii Telescope on the nights of 1991 July 19-21. These observations were made using a $120^{\prime \prime} \times 1^{\prime \prime}$ slit and the 1200 line $\mathrm{mm}^{-1}$ grating in the Hertzberg spectrograph, resulting in a spectral resolution of $1 \AA$ (FWHM). Foreground field stars happened to lie along the major and minor axes of the galaxy and allowed us to align the slit precisely from exposure to exposure. Neither of the stars turned out to have unusual spectra. Because of the large radius of this galaxy, the nucleus had to be aligned near one end of the slit in order to allow an adequate measurement of the sky. The position angles quoted above show the direction along which more radius was sampled. The spectra cover the wavelength range 4650-5650 $\AA$; most of the information that we use to extract the LOSVDs comes from the $\mathrm{Mg} \mathrm{b}$ feature at $5190 \AA$, although a number of weaker iron lines also contribute to the total signal. Standard IRAF routines were used to flat-field and wavelength-calibrate the data into 2048 logarithmically spaced wavelength bins (one [ $\ln \lambda]$-bin corresponds to $28.5 \mathrm{~km} \mathrm{~s}^{-1}$ ). Adjacent spectra were grouped and averaged along the slit until a signal-to-noise ratio of 20 was achieved everywhere (except in the outer regions of the disk, where uncertainties in the sky subtraction limited the signal-to-noise ratio to 10 ) and the continuum light level was subtracted from these data. The central 1500 wavelength bins of the co-added spectra were then passed through the UGD algorithm (Paper I) in order to recover the full
LOSVD at different positions in the galaxy. The UGD analysis of these data was made using 15 unresolved Gaussian components with dispersions of two bins and means at bins 14,17 , $\ldots, 53$ and 56 .

The continuum-subtracted spectra and the best-fit to these data produced by the UGD algorithm are shown in Figure 3. The models provide adequate fits to the data, although at some radii the model spectral lines are a little shallower than they appear in the data. We have verified that this discrepancy is only marginally a consequence of the smoothness requirement on the LOSVD and that is it instead due in large measure to template mismatch (which is discussed further in $\S 3$, where it is shown that this effect does not compromise the derived LOSVDs). The corresponding line-of-sight velocity distributions as a function of radius are presented in Figures 4 and 5: the surface plots of Figure 4 give an overall impression of the shapes of the velocity distribution at different radii, while the percentile plots in Figure 5 allow a more quantitative examination of the LOSVDs. As expected for an axisymmetric system, the major axis LOSVDs appear antisymmetric about the nucleus (most stars orbiting the galaxy are seen approaching on one side, receding on the other), and on the minor axis, the modal velocities are independent of position and the profiles are quite symmetric. Note that these symmetries are in no way imposed upon the method, and so the consistency of the results obtained from independent spectra on opposite sides of the galaxy gives a first indication that the UGD algorithm is returning a good approximation to the true LOSVD. The fact that adjacent spectra return very similar LOSVDs provides further confidence in the method.

Apart from these reassuring symmetries, the most striking feature in the derived LOSVDs is the long tail away from the peak rotation velocity on the major-axis, which is apparent out to large radii. This tail extends through the systemic velocity to retrograde orbits; as can be seen from the percentiles in Figure $5 a$, about $30 \%$ of the starlight in the disk consistently appears to be counterrotating relative to the majority of the stars. Figure 6 presents the individual LOSVDs along the majoraxis, which show definite indications of a secondary peak in the velocity distribution at the negative circular speed. This secondary peak suggests that the counterrotating stars have a small velocity dispersion and that they hence constitute a retrograde disk population.

\section{TESTS OF THE RESULTS}

Before we look further into the dynamical implications of this surprising discovery, it is important to ascertain that we are not being misled by some nonastrophysical phenomenon which might cause the UGD algorithm to generate spurious velocity distributions. In this section, we therefore examine whether the apparent counterrotation in NGC 7217 could be explained by such mundane systematic effects.

The UGD method has been tested extensively using simulated data. Several such tests, based on data at slightly lower resolution obtained with the Multiple Mirror Telescope, are described in Paper I. In these tests, we selected a known broadening function, convolved it with a stellar spectrum, added noise to the simulated data, and analyzed the resulting fake spectrum with the UGD algorithm. These tests have shown that, for data at a reasonable signal-to-noise level, the method consistently returns a reasonable representation of the input LOSVD and that the error bars generated by the algorithm are a realistic measure of the uncertainty in the LOSVD 


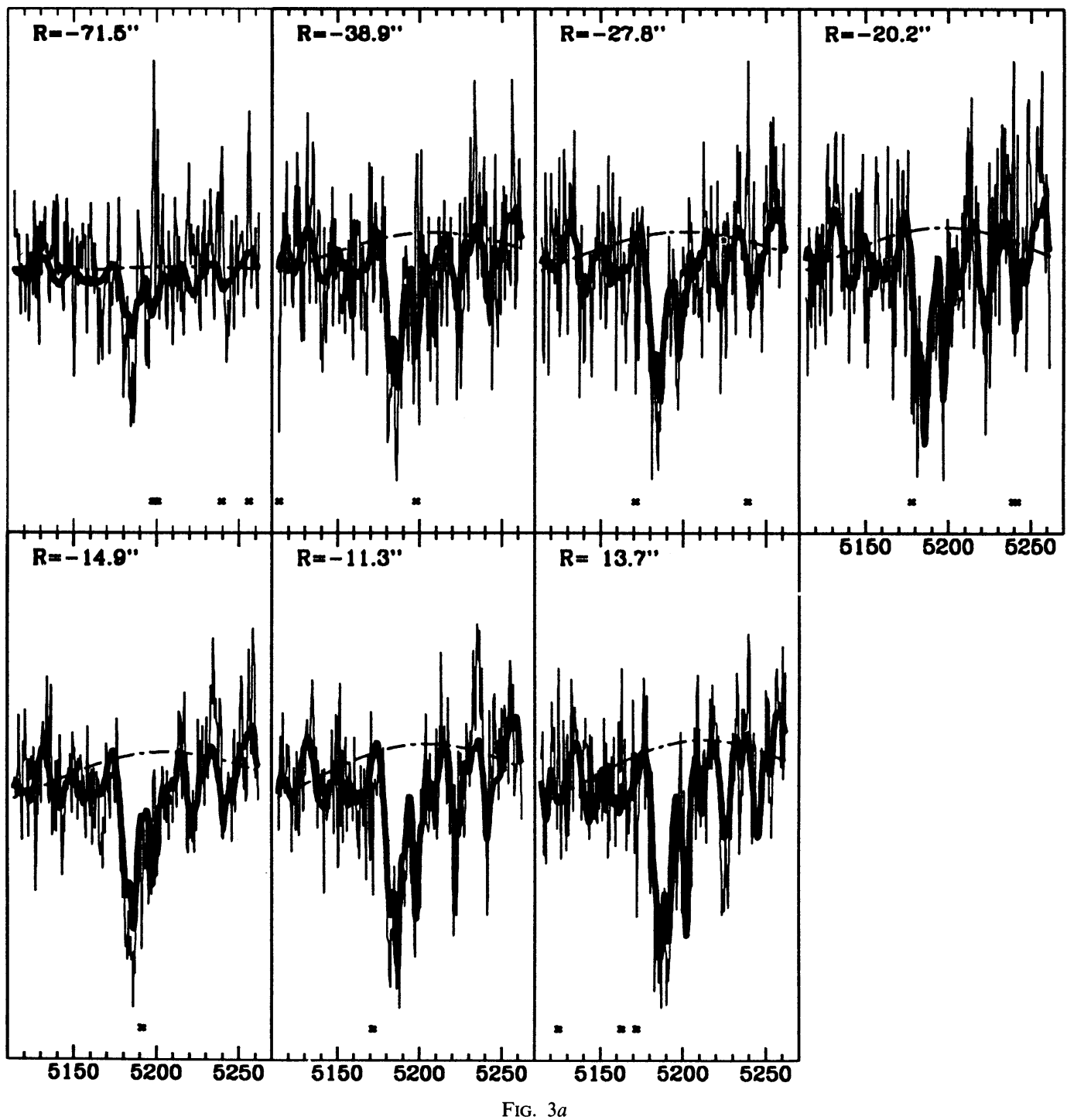

Fig. 3.-Central 300 pixels of the absorption-line spectra (centered on the dominant $\mathrm{Mg} b$ feature) used to determine the LOSVDs along the major and minor axes at radii exceeding $10^{\prime \prime}$. The convolutions of a continuum-subtracted stellar template spectrum with the best-fit LOSVDs returned by the UGD algorithm are shown as heavy lines. The residual continua in these spectra, which were also fitted by the UGD analysis (see Paper I), are shown as a dot-dashed curve. Crosses indicate those pixels which were rejected after a $3 \sigma$ clipping iteration. (a) Major-axis spectra (radius is positive east of the nucleus). (b) Minor-axis spectra (radius is positive south of the nucleus). The feature near $5200 \AA$ in the last three spectra arose from a bad column in the CCD and was excluded from the fits.

(although it must also be remembered that the errors on adjacent parts of the velocity distribution are significantly correlated).

In view of the peculiarity of the results that we find for NGC 7217, we have repeated several of the tests on the UGD algorithm using simulated data which closely resemble the observed major-axis spectra. As an input LOSVD we chose the sum of two Gaussians, similar to the velocity distribution that we found in the disk of NGC 7217: $F\left(v_{\text {los }}\right)=0.7 N(4.9,2.8)$ $+0.3 N(-4.9,2.8)$, where $N(\mu, \sigma)$ is a unit-integral Gaussian of mean $\mu$ and dispersion $\sigma$ bins. A spectrum of the G9III star HD 6, which we obtained with the same setup and processing sequence that was used for the NGC 7217 observations, was convolved with the velocity distribution, and varying amounts of noise were added. The resulting spectra were then analyzed using the UGD algorithm with the same set of unresolved
Gaussian components as were employed in the analysis of NGC 7217.

Figure 7 shows the deconvolution of the convolved spectrum with no noise added. Even though the basis functions do not have the same dispersion or means as the two components that make up the LOSVD, the input LOSVD is recovered accurately. This example illustrates the generality with which unresolved Gaussian basis functions can reproduce smooth LOSVDs.

The effects of noise are illustrated in Figure $8 a$, where we show the scatter in the LOSVDs derived from 100 realizations of the convolved spectrum, degraded until they had a signal-tonoise ratio of 20 in each bin (similar to the quality of data in the co-added spectra of NGC 7217). The mean profile agrees well with the input LOSVD, and the error bars returned by the algorithm (see Paper I) provide a realistic handle on the scatter 


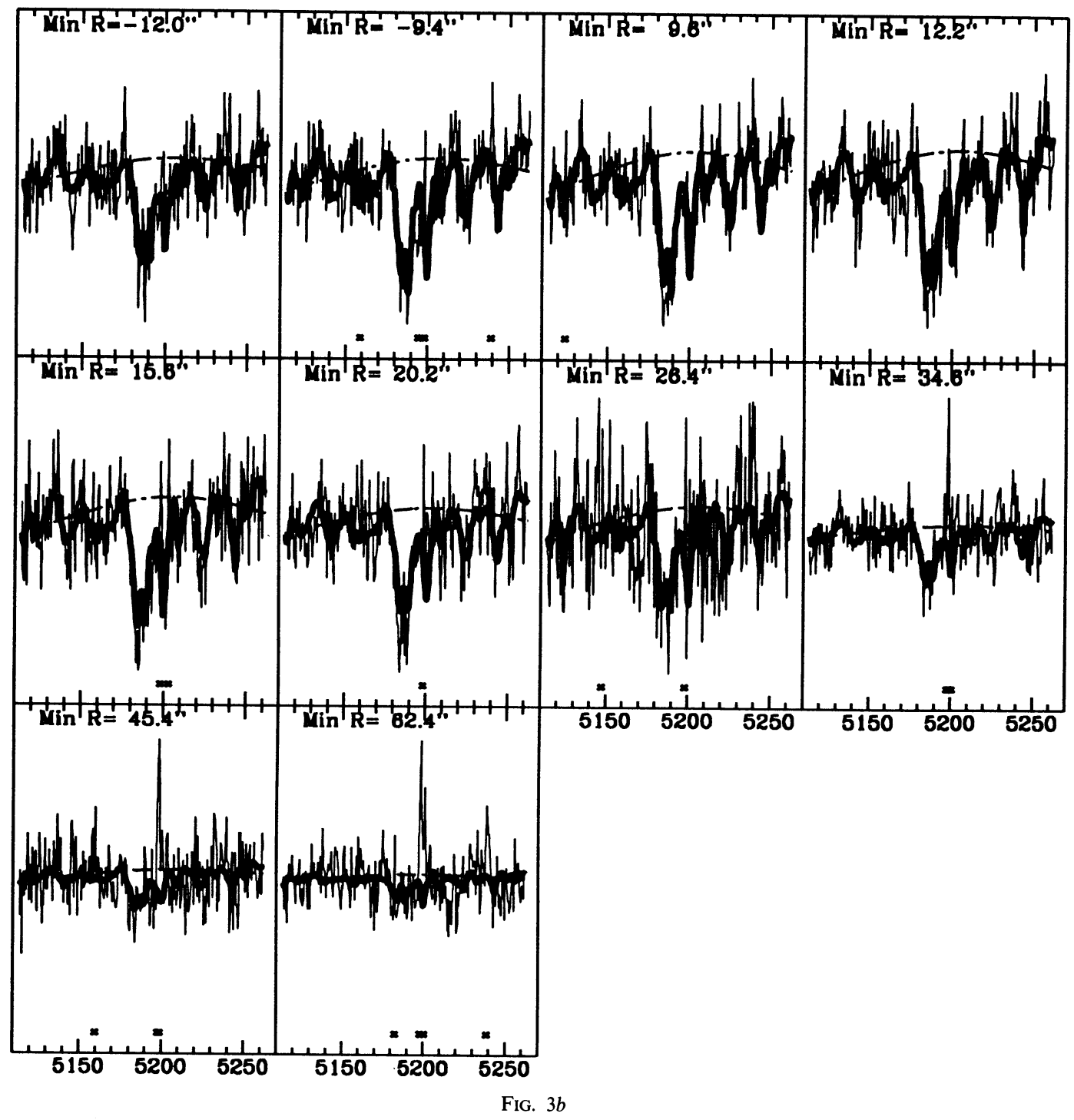

between the different realizations. A similar plot for a signal-tonoise ratio of 10 is shown in Figure $8 b$; even for the lower signal-to-noise level, the main features of the velocity disribution are preserved.

In these simulations, we have modeled the galaxy spectrum as the convolution of a single stellar template with a velocitybroadening function. In reality, of course, galaxies contain stars of many different spectral types. The assumption that a galaxy can be modeled using a single stellar type is partly justified because the light of a galaxy is dominated by $\mathrm{K}$ giant stars with similar spectra. Nevertheless, it is sensible to ask whether a single template can be sufficiently representative of the luminosity-weighted stellar mix in the galaxy spectrum for reliable LOSVDs to be extracted. We can investigate the effects of mismatch between the spectra of the galaxy and the template star by creating artificial galaxy data from the broadened spectra of stars with a range of spectral types. We can then apply the UGD algorithm to these spectra using a single stellar template spectrum and compare the recovered LOSVDs. Figure 9 presents the results of such an analysis which show that the derived LOSVD only depends very weakly on the effective spectral type of the galaxy and that template mismatch does not significantly distort any asymmetric tails in the LOSVD. Further tests in which the different stellar spectra were convolved with a single Gaussian before passage through the UGD algorithm were never found to yield the strong velocity distribution asymmetries found in NGC 7217, and so we can rule out template mismatch from giant-branch stars as a possible cause of spurious secondary peaks in the LOSVD. We reached a similar conclusion in Paper I based on simulations of Multiple Mirror Telescope data, and Rix \& White (1992) also found that template mismatch was not a significant problem when deriving LOSVDs using an optimal extraction technique.

On the basis of these tests, the UGD analysis appears to be sound, and it is hard to see how the counterrotating component in the LOSVD can be attributed to the nonastrophysical sources of error described here. In particular, the residuals due to noise are well below the level of significance of the counterrotating population, and, in any case, such noise effects would not be expected to produce the consistent LOSVDs that we find from adjacent spectra. In addition, the 

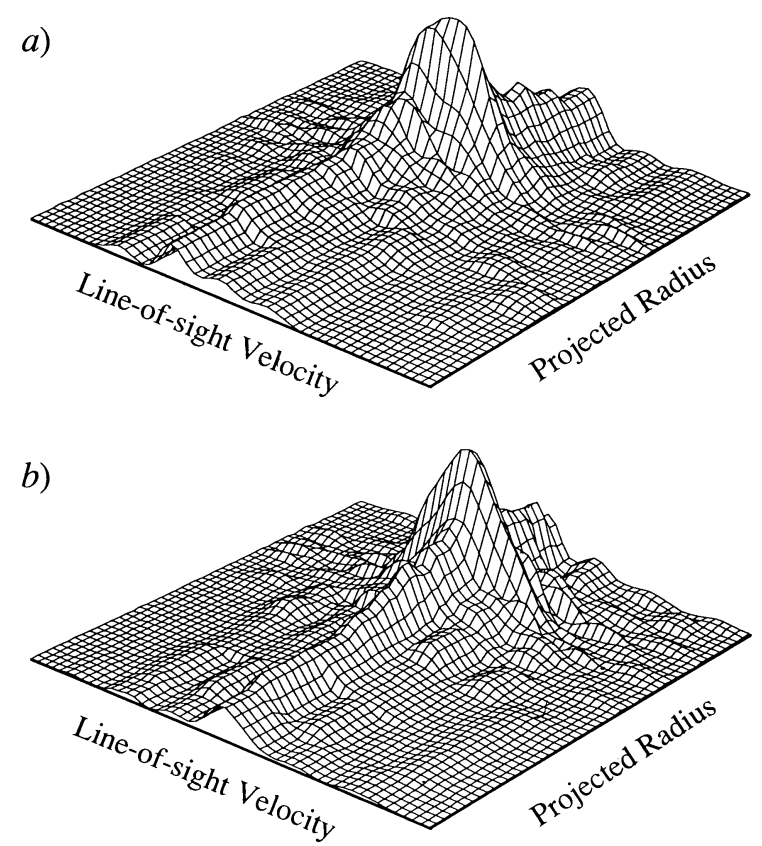

FIG. 4.-Surface plot of the line-of-sight velocity distributions derived from long-slit spectra of NGC 7217 using the UGD algorithm. The height of the surface indicates the density of stars at a particular projected radius and moving with a particular line-of-sight velocity. The grid lines are separated by $1^{\prime \prime}$ on the spatial axis and $28.5 \mathrm{~km} \mathrm{~s}^{-1}$ on the velocity axis. In order to increase the dynamic range, the LOSVD at every radius was normalized by the square root of its integral before plotting. (a) Major-axis LOSVDs. (b) Minor-axis LOSVDs.

simulations show that template mismatch is not likely to produce systematic effects of the size necessary to explain the observed counterrotation.

Ultimately, it is almost impossible to come up with any sources of systematic error which might reproduce the features that the UGD algorithm finds in the LOSVDs. Any systematic

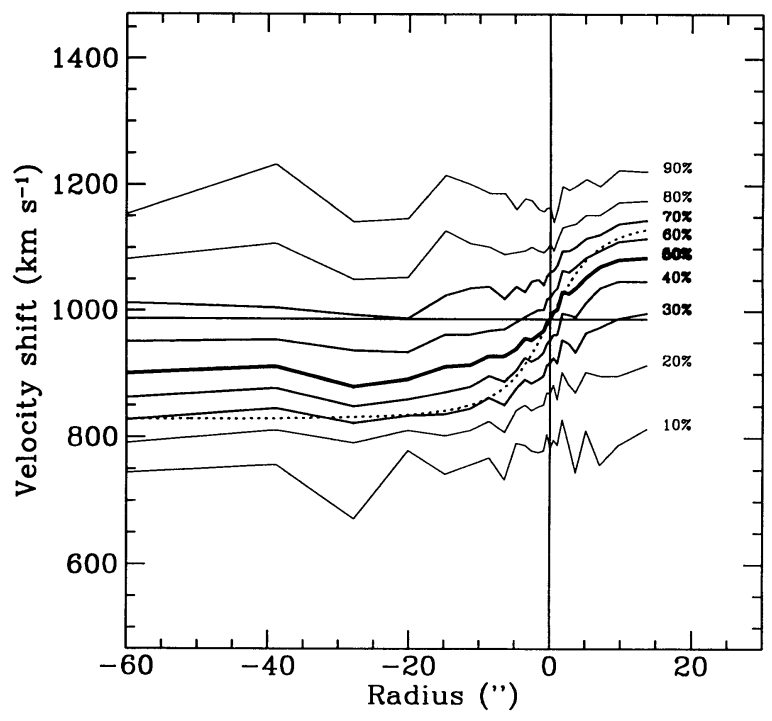

Fig. $5 a$ effect would have great difficulty explaining the major-axis results where there is a low-velocity tail on one side of the nucleus and a high-velocity tail on the other side of the nucleus, with both sets of LOSVDs derived from the same long-slit spectra. Furthermore, this systematic error must be absent from our analysis of similar spectra from the minor axis since here the derived velocity distributions are entirely symmetric. Finally, we note that the results obtained from majoraxis spectra of other disk galaxies using the same method show no signs of a counterrotating component (Kuijken \& Merrifield, in preparation).

\section{THE NATURE OF THE COUNTERROTATING COMPONENT}

Having established the reality of the very broad velocity distribution at all radii in NGC 7217 and the significant retrograde component in this distribution, we now seek a dynamical interpretation of these results. The most exciting possibility is that we are seeing a counterrotating population of stars in the disk of the galaxy. However, there are a number of other possible explanations for the shape of the LOSVDs which must also be considered :

1. Radial projection along the line of sight.-A galactic disk is not infinitely thin, and so any line of sight samples the kinematics of the system over a range of radii, broadening the observed velocity distribution. However, NGC 7217 is only inclined $35^{\circ}$ from face-on; typical ratios of vertical to radial scale lengths in a disk are about 1:10, and so the radial averaging implied by line-of-sight projection is likely to be minimal in this case.

2. Broadening by vertical motions.-Because of the relatively face-on orientation of NGC 7217, the vertical motions in the galaxy's disk project favorably into the LOSVD. A high vertical velocity dispersion would certainly have the effect of broadening the major-axis LOSVD, possibly creating a significant wing of apparently counterrotating stars. However, for a disk in equilibrium, the vertical velocity distribution must be symmetric, and so a significant wing toward faster rotation

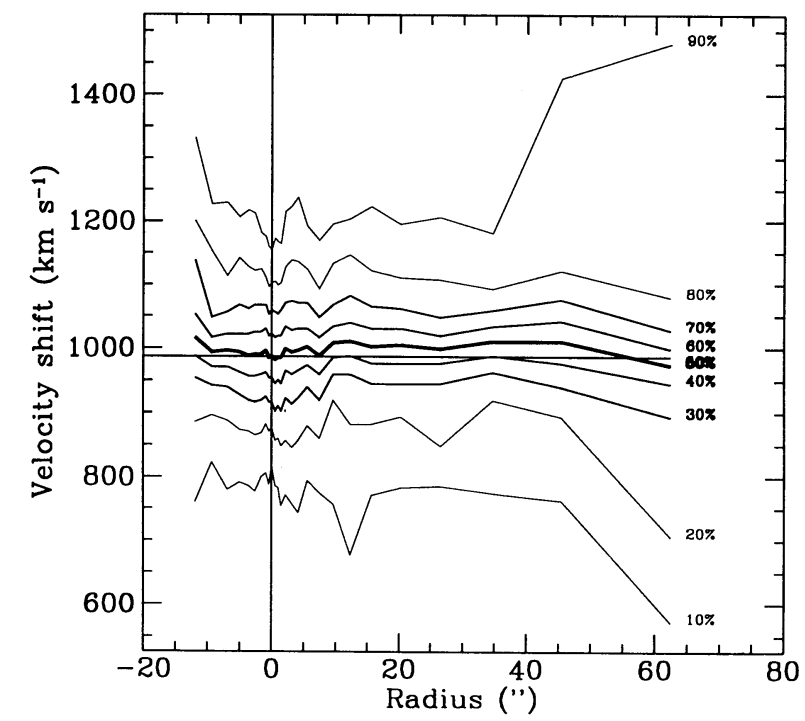

Fig. $5 b$

Fig. 5.-Line-of-sight velocity distributions presented as cumulative velocity distributions ( $90 \%$ of the stars have velocity below the line labeled $90 \%$, and so on). The sign of $R$ is as in Fig. 3. (a) Major-axis percentiles. The emission-line rotation curve (Peterson et al. 1978) is shown as a dotted line; it closely traces the peaks of the LOSVD. (b) Minor-axis percentiles. 


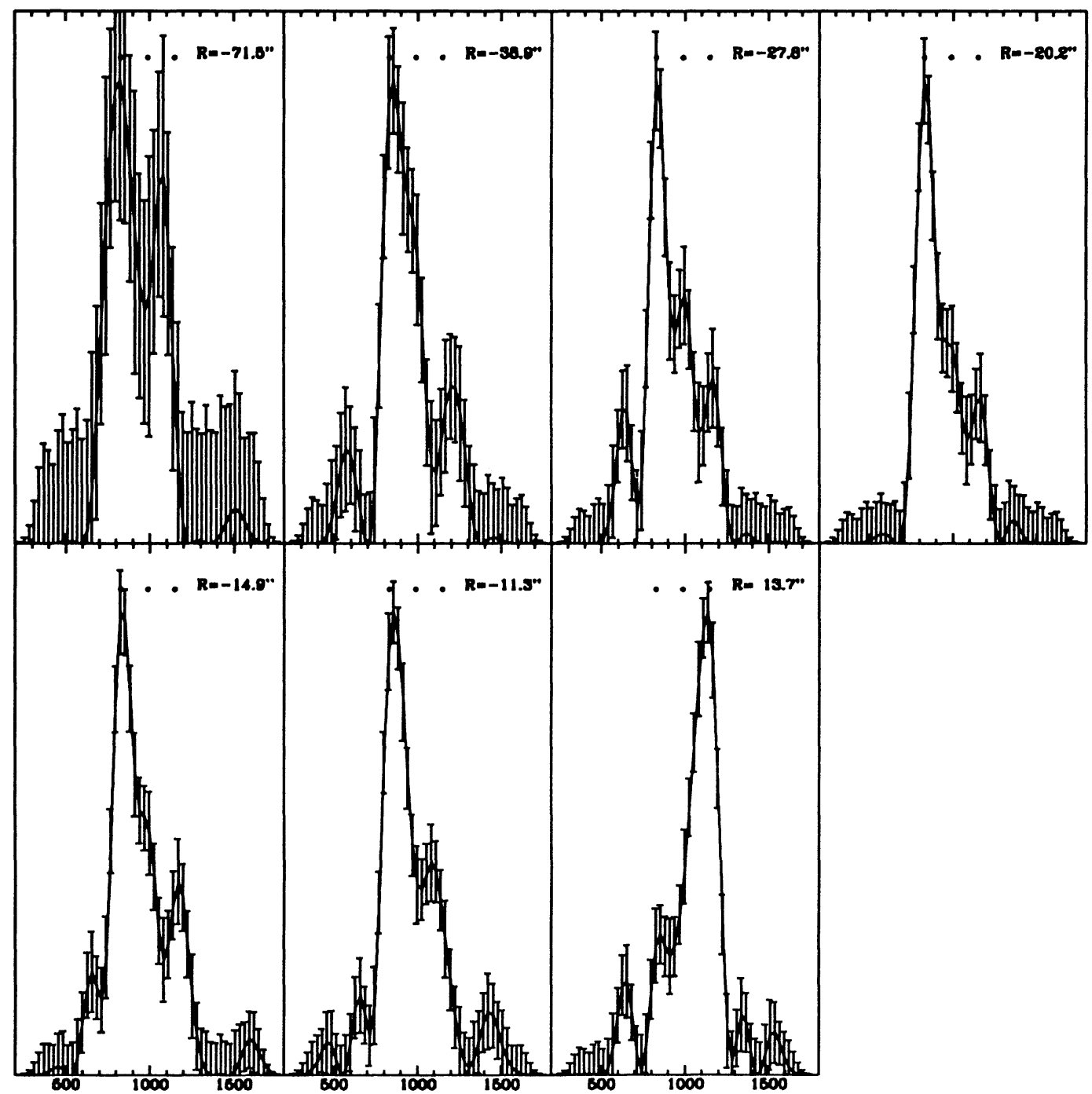

Fig. 6. - LOSVDs for the major-axis disk spectra $\left(|R|>10^{\prime \prime}\right)$. The sign of $R$ is as in Fig. 3. Error bars for adjacent velocity bins are correlated, but they still represent the $1 \sigma$ uncertainty in the LOSVD at each velocity (see Paper I). The three dots above the LOSVDs indicate the systemic velocity of NGC 7217 and the speeds of prograde and retrograde circular rotation.

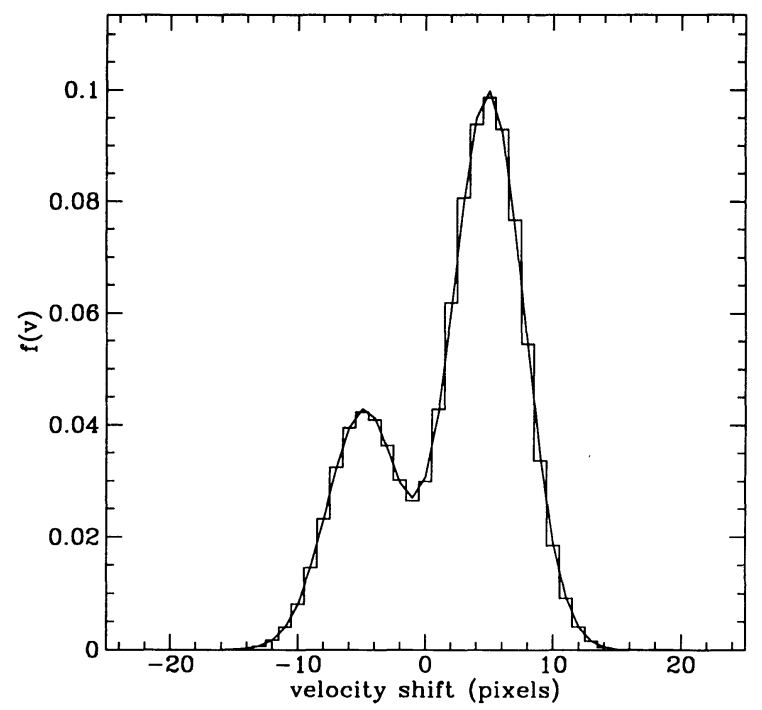

Fig. 7.-Comparison between a double-Gaussian model LOSVD (smooth curve) and the LOSVD which the UGD algorithm recovers when applied to a spectrum of a gG9 star convolved with this model (histogram). should also result. As can be seen in Figure 6, the line profiles are very skew, and there is no evidence for such a high-velocity wing. The extent to which the LOSVDs are broadened by vertical motions is limited by the steep high-velocity side of the profiles, and clearly this effect is not sufficient to cause a large, apparently retrograde wing.

3. Bulge contamination.-A more plausible explanation for the long counterrotating tail is that it is really part of the velocity distribution of a large bulge. The observed LOSVD is then the sum of a disk distribution function with a fairly narrow peak near the circular speed, and a large-dispersion bulge distribution with very little net rotation. Although such a model could provide an adequate description of the LOSVD at small radii, it does not fit with the data further from the center of the galaxy. While the bulge-to-disk ratio is expected to decline rapidly with radius, the fraction of the LOSVD on the counterrotating side remains quite constant outside the central $10^{\prime \prime}$ (see Fig. 5a), which suggests that this component is not associated with the bulge. Direct evidence that the bulge is not a major contributor to the total luminosity at larger radii comes from the brightness profile along the major axis (see Fig. 2) which shows a distinct break near 10", beyond which it 


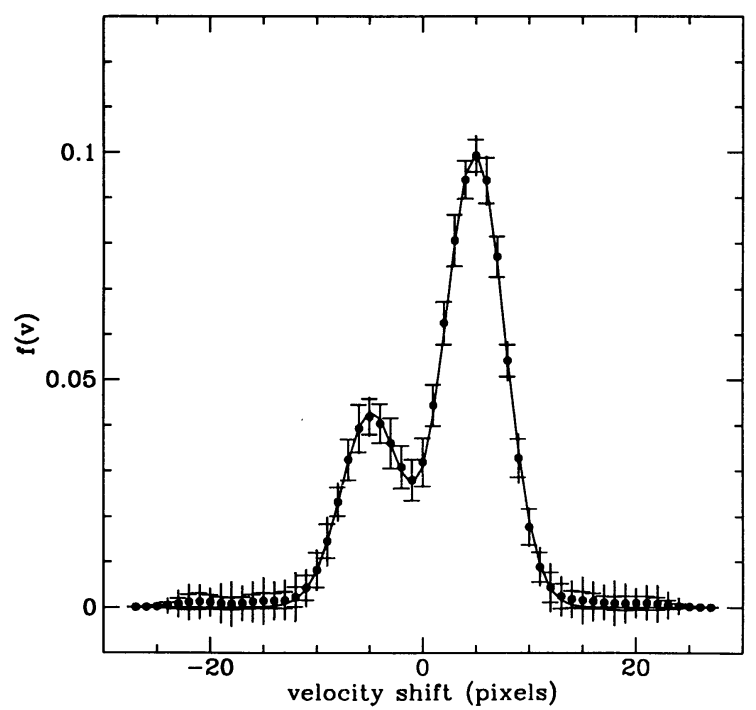

FIG. $8 a$

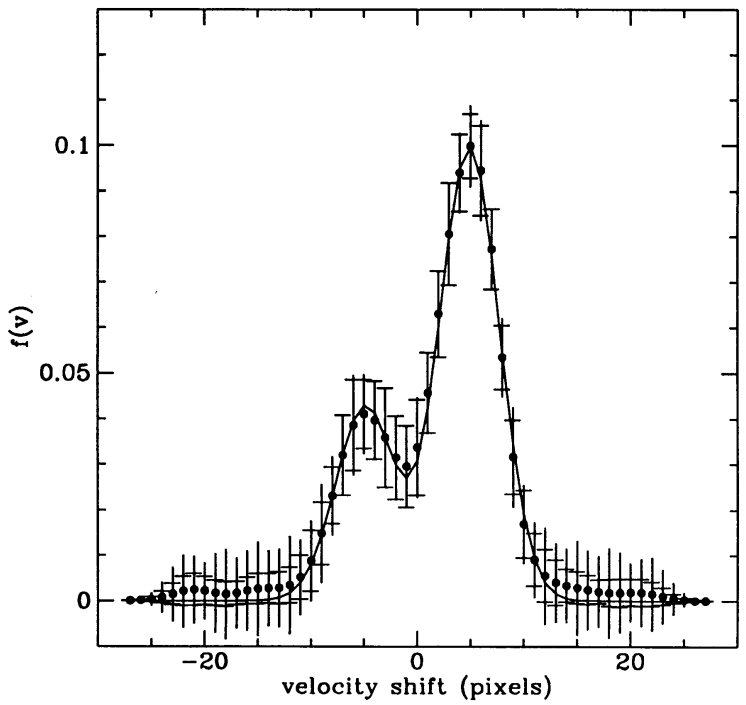

FIG. $8 b$

FIG. 8.-Simulations of the effects of photon noise in recovering a known LOSVD. The solid line illustrates the input LOSVD, which was convolved with a

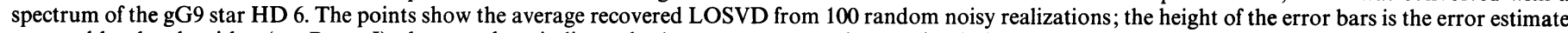

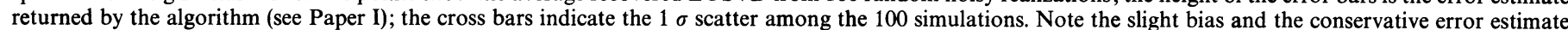

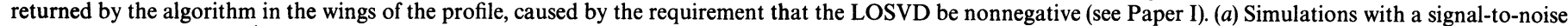
ratio of 20 per $28.5 \mathrm{~km} \mathrm{~s}^{-1}$ bin. (b) Simulations with a signal-to-noise ratio of 10 per $28.5 \mathrm{~km} \mathrm{~s}^{-1} \mathrm{bin}$.

follows the exponential form that would be expected if the disk light were dominant. Furthermore, if the $30 \%$ of counterrotating starlight is to be attributed to the bulge, then the whole bulge must be responsible for at least $60 \%$ of the observed luminosity at all radii, and this fraction is very large for a disk galaxy which displays (albeit weak) spiral structure.

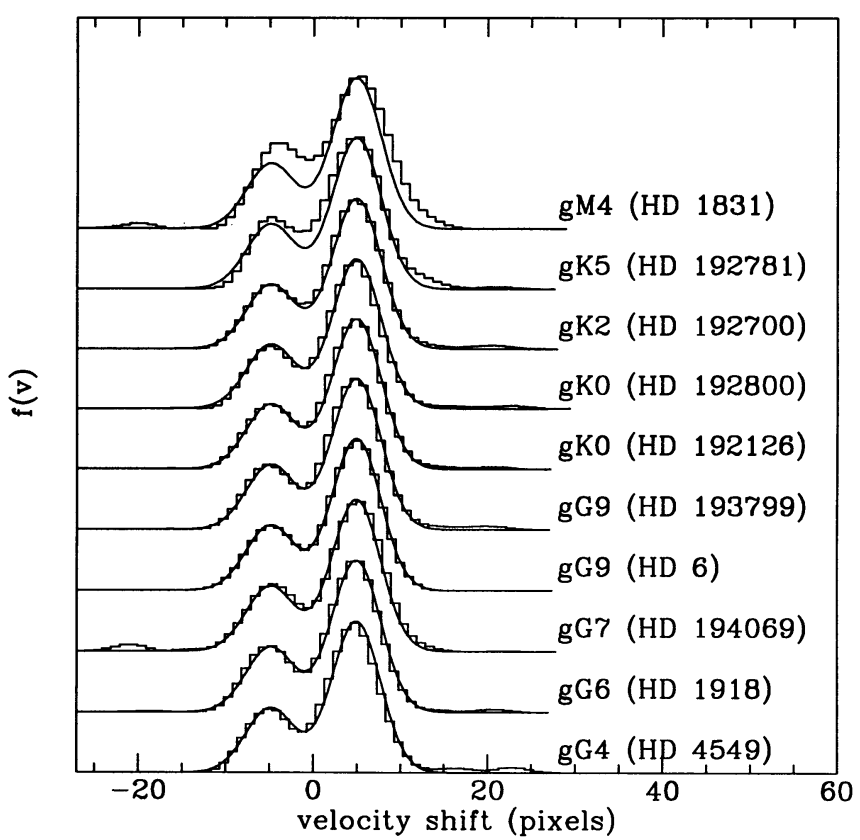

FIG. 9.-Simulations of the effects of template mismatch on the derived LOSVDs. Ten different spectra with types ranging from gG4 to gM4 were convolved with the input LOSVD (smooth curve), and the UGD algorithm was then used to recover the LOSVD (histogram) using the gG9 star HD 6 as a template spectrum. The output LOSVDs have been shifted and rescaled to allow for differences in radial velocity and line strength. Only for very-latetype spectra does template mismatch become a significant problem.
Finally, there are consistent indications of a secondary peak in the major-axis LOSVD near the negative circular speed (see Fig. 6) -in each of the independent LOSVDs the velocity distribution at this velocity is at least $3 \sigma$ above zero. Such a peak would not occur in a broad bulge velocity component and its presence indicates the existence of a relatively cold counterrotating disk population.

Thus we see that none of these kinematic effects can provide a plausible explanation for more than a small fraction of the $30 \%$ of apparently counterrotating stars in NGC 7217. Instead, we are forced to return to the hypothesis that the observed counterrotating component is made up of stars on retrograde orbits in the disk of the galaxy.

\section{DYNAMICAL DISK MODELS}

As we have seen in the previous section, it is likely that the LOSVDs presented in $\S 2$ are dominated by disk light outside the central $10^{\prime \prime}$. We can hence go beyond the purely kinematic description of the data in this region to see if we can adequately describe the observations using a full dynamical disk model. Such modeling will allow us to look in more quantitative detail at the apparent counterrotation in the LOSVDs, and to check that these observations are consistent with a dynamical system in equilibrium. Direct dynamical modeling of the spectra makes the fullest possible use of the data, by incorporating fundamental dynamical constraints in the models. This approach is most practical if the galaxy has a fairly simple geometry with a well-understood orbital structure. In order to see how we can fit a detailed model to our data from this galaxy, we must first consider the observable dynamical properties of stellar disks in general.

If a galaxy is axisymmetric and in equilibrium, then the orbits in its potential are governed by three integrals (i.e., constants) of motion. If, for the moment, we ignore the thickness of the galaxy's disk and motions in the direction perpendicular to the plane (the $z$-direction), then all the orbits are 
described by just two integrals of motion: the angular momentum about the axis of symmetry, $L=R v_{\phi}$, and the energy of motions in the disk plane, $E_{p}=(1 / 2) v_{R}^{2}+(1 / 2) v_{\phi}^{2}+\Psi(R)$. As long as we have a $21 \mathrm{~cm}$ or optical emission-line rotation curve, $v_{c}(R)$, the gravitational potential in the disk plane, $\Psi(R)$, is observationally determined via the relation $v_{c}^{2}(R)=$ $R d \Psi / d R^{1}$

The distribution function (DF) $f$ is a function of the phasespace coordinates only via the integrals of motion (Jeans's theorem): $f\left(R, v_{R}, v_{\phi}\right) \equiv f\left(E_{p}, L\right)$. Integrating $f$ over $v_{R}$ or $v_{\phi}$ produces the velocity distributions of, respectively, $v_{\phi}$ or $v_{R}$ at radius $R$ :

$$
\begin{aligned}
f_{\phi}\left(R, v_{\phi}\right) & =\int_{-\infty}^{\infty} f d v_{R}=2 \int_{W_{\phi}}^{\infty} \frac{f\left(E_{p}, L\right) d E_{p}}{\left[2\left(E_{p}-W_{\phi}\right)\right]^{1 / 2}} \\
& \equiv f_{\phi}\left(W_{\phi}, L\right), \\
f_{R}\left(R, v_{R}\right) & =\int_{-\infty}^{\infty} f d v_{\phi}=R^{-1} \int_{-\infty}^{\infty} f\left(W_{R}+\frac{1}{2} L^{2} / R^{2}, L\right) d L \\
& \equiv f_{R}\left(W_{R}, R\right),
\end{aligned}
$$

where $W_{R}=\Psi(R)+(1 / 2) v_{R}^{2}$ and $W_{\phi}=\Psi(R)+(1 / 2) v_{\phi}^{2}$.

If such a two-dimensional disk is observed at an inclination $i$ to the line of sight, then the major- and minor-axis LOSVDs at a projected distance $R_{\text {proj }}$ from the nucleus are just rescaled versions of $f_{\phi}$ and $f_{R}$ :

$$
\begin{gathered}
F_{\text {maj }}\left(R_{\text {proj }}, v_{\text {los }}\right) \equiv f_{\phi}\left(R=R_{\text {proj }}, v_{\phi}=v_{\mathrm{los}} / \sin i\right) / \sin i, \\
F_{\text {min }}\left(R_{\mathrm{proj}}, v_{\mathrm{los}}\right) \equiv f_{R}\left(R=R_{\mathrm{proj}} / \cos i, v_{R}\right. \\
\left.=v_{\mathrm{los}} / \sin i\right) /(\sin i \cos i),
\end{gathered}
$$

where we have subtracted out the systemic redshift of the galaxy from the line-of-sight velocity. The factors of $\sin i$ and cos $i$ arise from the Jacobian of the transformations from $R$ and $v$ to $R_{\text {proj }}$ and $v_{\text {los }}$.

Equation (3) means that $f_{\phi}\left(R, v_{\phi}\right)$ and $f_{R}\left(R, v_{R}\right)$ are observable quantities. The same is true of $W_{\phi}$ and $W_{R}$ (they are simply reparametrizations of $R_{\text {proj }}$ and $\left.v_{\text {los }}\right)$, and hence $f_{\phi}\left(W_{\phi}, L\right)$ and $f_{R}\left(W_{R}, R\right)$ are also observable. Since equation (2a) is an Abel integral of the DF in the variable $E_{p}, f_{\phi}\left(W_{\phi}, L\right)$ can be uniquely inverted to yield $f\left(E_{p}, L\right)$. In other words, the LOSVD on the major-axis of a thin disk determines the full DF of the motions within the system. It therefore tells us all we need to know in order to predict the result of any other dynamical measurement in the system. In particular, in a thin disk with no vertical motions the major-axis LOSVD fully determines the minoraxis LOSVD.

In fact, there is yet more redundancy in the observable properties of a thin disk. The transformation from $\left(R, v_{\phi}\right)$ to $\left(W_{\phi}, L\right)$ in equation (2a) is not one-to-one, but two-to-one: every point $\left(W_{\phi}, L\right)$ corresponds to two $\left(R, v_{\phi}\right)$-values. It is straightforward to show that the two $\left(R, v_{\phi}\right)$ pairs are the inner and outer radius and azimuthal speed of an orbit with energy $W_{\phi}$ and angular momentum $L$. At the inner point $\left|v_{\phi}\right|>v_{c}$, while at the outer point $\left|v_{\phi}\right|<v_{c}$. The distribution function is therefore completely specified both by the part of $F_{\text {maj }}\left(R_{\text {proj }}\right.$, $v_{\text {los }}$ ) where $\left|v_{\text {los }}\right|>v_{c}\left(R_{\text {proj }}\right) \sin i$, and by the part where $\left|v_{\text {los }}\right|<$ $v_{c}\left(R_{\text {proj }}\right) \sin i$.

This analysis suggests a practical approach to fitting a quasi-

${ }^{1}$ In the case of NGC 7217, Peterson et al. (1978) have obtained an emission-line rotation curve which, after deprojection, is well-fitted by the simple relation $v_{c}(R)=R^{2}\left[R^{2}+\left(7^{\prime \prime}\right)^{2}\right]^{-1 / 2} \times 280 \mathrm{~km} \mathrm{~s}^{-1}$. self-consistent dynamical model to our spectra of the disk of NGC 7217. If the motions perpendicular to the plane of the galaxy are negligible, then we can search for the function $f\left(E_{p}, L\right)$ that results in LOSVDs $F_{\text {maj }}$ and $F_{\text {min }}$ which, when convolved with a stellar template, best reproduce the observed spectra of the galaxy. Because the planar DF is overdetermined by the observed properties, significant motions perpendicular to the plane of the galaxy will prevent this method from producing a perfect fit to the observations. We can try to improve the fit by adding further broadening to the LOSVDs to model the velocity distribution in the $z$-direction, and thus we can also constrain the nonplanar motions in the system. The application of this approach to the NGC 7217 data is described in the next section.

\section{THE DISTRIBUTION FUNCTION OF NGC 7217}

\subsection{Quadratic Programming}

The only outstanding problem in fitting a dynamical model to the spectral data is how to go about finding the best-fit planar DF, $f\left(E_{p}, L\right)$. The method that we adopt is essentially that which Dejonghe (1989b) used in his study of M87; we write the DF as a linear combination of (analytic) components with unknown coefficients which we solve for by least-squares fitting to the data. The principal difference between our analysis and that of Dejonghe is that, whereas he fitted the models to velocity moment data (which were, in fact, derived using single-Gaussian fitting of absorption-line profiles and are therefore subject to the systematic uncertainties mentioned in the introduction), we fit to the observed spectra themselves by broadening a stellar template with the LOSVD of each DF component. Thus we make full use of all the kinematic information in our observations. We believe that this analysis represents the first such direct fit of a stellar dynamical model to a spectral data set.

The principle behind a reconstruction of the DF using "building block" components, $f_{\alpha}\left(E_{p}, L\right)$, is the same as for the one-dimensional reconstruction of the LOSVD from unresolved Gaussian components, which is described in detail in Paper I. Essentially, we just write

$$
f\left(E_{p}, L\right)=\sum_{\alpha} x_{a} f_{\alpha}\left(E_{p}, L\right)
$$

and vary all the $x_{\alpha} \mathrm{s}$ in order to minimize in a least-squares sense the difference between the observed spectra and the prediction of the model DF. For the case at hand, we have observed absorption-line spectra along different lines of sight $l$, $S_{l}\left(\ln \lambda_{j}\right)$, with errors $\sigma_{j l}$ on each wavelength bin. The DF component $f_{\alpha}$ generates a LOSVD $F_{\alpha l}(v)$ for the line of sight $l$ through the galaxy and therefore produces a component of the total spectrum along this line of sight, $S_{\alpha l}(\ln \lambda)$, which is the convolution of $F_{\alpha l}(v)$ with the stellar template spectrum. The best-fit solution for the DF is hence found by mimimizing the quantity

$$
\chi^{2}\left(x_{\alpha}\right)=\sum_{j l}\left[\frac{S_{l}\left(\ln \lambda_{j}\right)-\Sigma_{\alpha} x_{\alpha} S_{\alpha l}\left(\ln \lambda_{j}\right)}{\sigma_{j l}}\right]^{2}
$$

with respect to the coefficients $x_{\alpha}$. The sum covers all the observed spectra, and thus the fit is made to all the data simultaneously. As was the case for UGD, this sum is a quadratic expression in the unknowns $x_{\alpha}$, and here, too, we have to minimize subject to linear constraints: the phase space density $f\left(E_{p}, L\right)$ must be nonnegative at all values $\left(E_{p}, L\right)$ that corre- 
spond to physical orbits. Thus we have a set of constraints of the form

$$
\sum_{\alpha} x_{\alpha} f_{\alpha}\left(E_{p k}, L_{k}\right) \geq 0 \text {. }
$$

In practice, a large but finite grid of such constraints is used, transforming the minimization into a quadratic programming problem (Dejonghe 1989a).

It is possible to think of many possible sets of building blocks. Because calculation of the Hessian matrix $D_{i k}$ (i.e., the quadratic coefficients of $\chi^{2}\left(x_{\alpha}\right)$ in eq. [5]; see also Paper I) requires convolution of the model LOSVDs with a stellar template, we have chosen ellipsoidal DF components whose velocity distributions are everywhere Gaussian. These LOSVDs can be convolved analytically with the template spectrum which speeds up numerical computations. We describe these elementary DFs and the resulting model for the disk of NGC 7217 below ( $(6.2)$. The analysis was also repeated using a less parametric set of building block DFs with a somewhat finer resolution in integral space in order to check that the results do not depend strongly on the choice of basis functions $(\S 6.3)$.

\subsection{Ellipsoidal Basis Functions}

Except for a normalization factor, the most general DF whose velocity distribution is everywhere Gaussian can be written as

$$
f_{\alpha \beta \gamma}\left(E_{p}, L\right)=\exp \left[-\beta \frac{E_{p}}{v_{c}^{2}}-\frac{1}{2} \gamma^{2}\left(\frac{L}{h v_{c}}-\alpha\right)^{2}\right] .
$$

In this equation, the constants $v_{c}$ (the asymptotic circular speed in the galaxy) and $h$ (the exponential scale length of the disk) are used to render the parameters $\alpha, \beta$, and $\gamma$ dimensionless, but they do not have any physical significance. $f_{\alpha \beta \gamma}$ is Gaussian in $L$, with mean $\alpha h v_{c}$ and dispersion $h v_{c} / \gamma$, and exponential in $E_{p}$, with scale length $v_{c}^{2} / \beta$. We have chosen to limit the "resolution" in $L$ by setting $\gamma=2$ for all components: since most orbits can be expected to be reasonably close to circular, this choice restricts us to DFs which do not vary sharply on scales below half an exponential scale length. The values of $\alpha$ must then be separated by at most $2 / \gamma=1$, so that the individual components are unresolved and we end up with a DF which varies smoothly with $L$. We used $\alpha=\{-3,-2,-1,0,1$, $2,3\}$, which suffices to cover prograde and retrograde orbits with home radii out to three exponential scale lengths. It turned out that only two $\beta$-values could be used: a denser grid resulted in components becoming too correlated, which caused problems for the quadratic programming algorithm. The values employed were $\beta=\{2.5,10\}$.

The velocity distribution due to a single component is a bivariate Gaussian in $v_{R}$ and $v_{\phi}$ : it is easily shown from equation (7) that

$$
f_{\alpha \beta \gamma}\left(R, v_{R}, v_{\phi}\right)=\frac{\Sigma(R)}{2 \pi \sigma_{R} \sigma_{\phi}} \exp \left[-\frac{1}{2} \frac{v_{R}^{2}}{\sigma_{R}^{2}}-\frac{1}{2} \frac{\left(v_{\phi}-\bar{v}_{\phi}\right)^{2}}{\sigma_{\phi}^{2}}\right],
$$

where

$$
\sigma_{R}=\frac{v_{c}}{\beta^{1 / 2}}, \quad \sigma_{\phi}=\frac{v_{c}}{\left[\beta+\gamma^{2}(R / h)^{2}\right]^{1 / 2}}, \quad \bar{v}_{\phi}=\frac{\alpha \gamma^{2}(R / h)}{\beta+\gamma^{2}(R / h)^{2}} v_{c},
$$

and

$$
\Sigma(R)=2 \pi \sigma_{R} \sigma_{\phi} \exp \left(-\beta \frac{\Psi+\frac{1}{2} \alpha^{2} \gamma^{2} \sigma_{\phi}^{2}}{v_{c}^{2}}\right)
$$

With these equations, it is a simple matter to calculate the (Gaussian) LOSVD for each component $f_{\alpha \beta \gamma}$ at every radius on the major and minor axes of NGC 7217 for which we have an absorption-line spectrum. The model absorption-line spectra are then just Gaussian convolutions of a stellar template. After calculating these component spectra, it is straightforward to set up the quadratic programming problem. One remaining difficulty is imposed by the constraint equations, as it takes many more points to cover the $\left(E_{p}, L\right)$-plane here than were required in the one-dimensional problem of extracting individual LOSVDs (Paper I), making it hard to force the algorithm to return a DF which is nonnegative everywhere. In practice, we set up a coarse grid of points at which the DF was forced to be positive, solved the quadratic programming problem, added extra constraints at points where the derived DF was negative, and repeated the process. This approach was found to yield a totally nonnegative DF after just one or two iterations.

We have applied this method to the major- and minor-axis spectra of NGC 7217 that were obtained from deprojected radii exceeding $10^{\prime \prime}$. The planar distribution function recovered by this analysis is presented in Figure 10. For clarity, this figure replaces the $E_{p}$-coordinate by $\left\{2\left[E_{p}-E_{\text {circ }}(L)\right]\right\}^{1 / 2}$, where $E_{\text {circ }}$ is the energy of the circular orbit of angular momentum $L$. This substitution has the effect of transforming the locus of circular orbits, which bounds the physical part of the integral plane, into a straight line. The new coordinate represents the radial velocity of a star on its orbit when it crosses its home radius (the radius of the circular orbit with the same angular momentum), so circular orbits lie along the lower boundary in this plane with eccentricity increasing upward. Figure 11 shows the intrinsic components of the velocity distribution at different radii corresponding to the DF in Figure 10.

It is immediately apparent from Figures 10 and 11 that the two-dimensional analysis confirms the presence of a sizeable counterrotating population. The true nature of this population is also revealed by these models since the analysis clearly places a discrete component at $v_{\phi}=-v_{c}$ (see particularly Fig. 11). Of course, we have now built in a dynamical "bias": all stellar orbits in an axisymmetric disk are oscillations about circular orbits, and so the circular speed assumes a special significance in the modeling. Nevertheless, there are plenty of building block DFs in our set which would have introduced stars at intermediate velocities (say, $v_{\phi}=(1 / 2) v_{c}$ ) at the cost of also

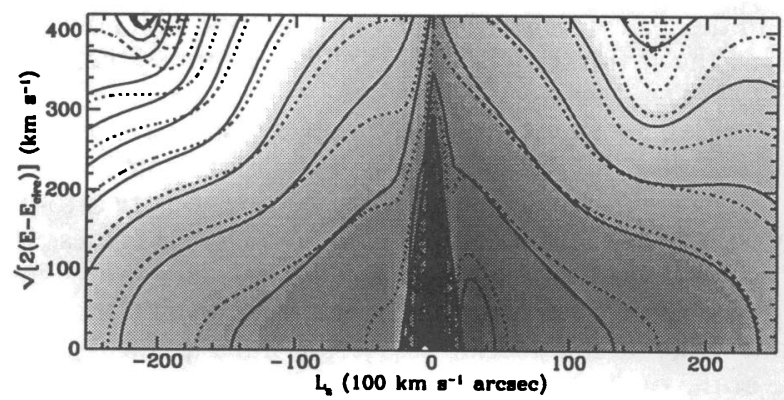

FIG. 10.-DF derived by fitting 14 ellipsoidal components, presented as a combined contour and gray-scale plot. The contours are separated by a factor of 4 in phase-space density. The hashed region contains those orbits which remain inside $R=10^{\prime \prime}$, therefore the DF is not constrained by our data in this part of phase space. The solid contours and gray scale show the DF recovered assuming that all the motions in the galaxy are planar; the dotted contours show the DF obtained assuming additional broadening due to vertical motions from a Gaussian velocity distribution with dispersion $\sigma_{z}=(100 \mathrm{~km}$ $\left.\mathrm{s}^{-1}\right) e^{-R / 60^{\prime \prime}}$. 

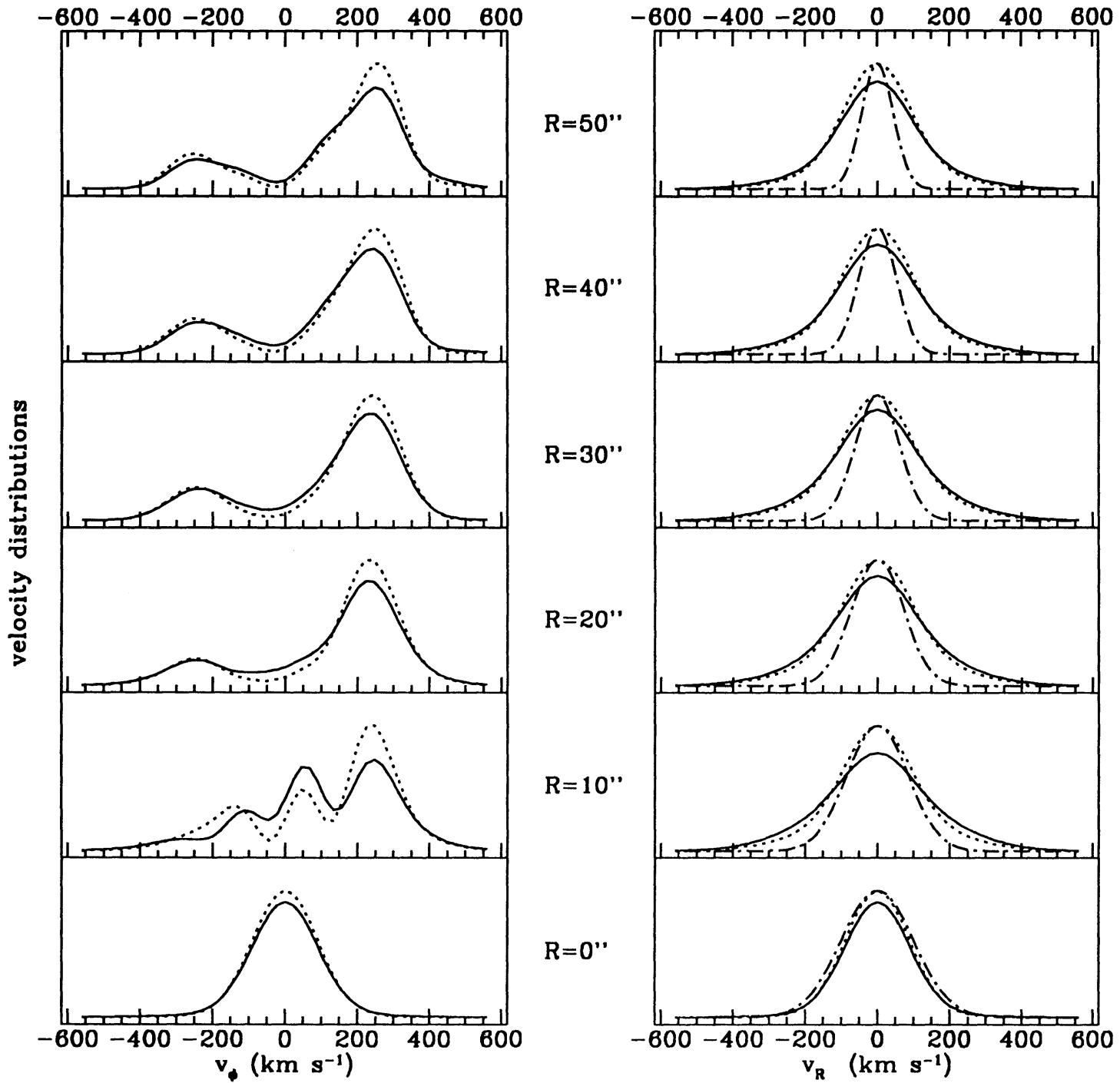

FIG. 11.- Individual components of the velocity distribution corresponding to the model DFs of Fig. 10. Solid lines show velocity distributions derived assuming that all the motions in the galaxy are planar; the dotted lines show velocity distributions derived assuming additional vertical motions due to a Gaussian velocity distribution with dispersion $\sigma_{z}=\left(100 \mathrm{~km} \mathrm{~s}^{-1}\right) e^{-R / 60^{\prime \prime}}$; the dash-dotted line (right-hand panels only) shows the velocity distribution in the $z$-direction for this latter case.

having stars with $v_{\phi}>v_{c}$ at smaller radii. The fit to the spectra implies that the amplitudes of these terms are small and confirms the presence of a secondary peak in the major-axis LOSVDs at the negative circular speed, as was suggested in $\S 2$. We can now see that the counterrotating stars constitute a kinematically distinct component rather than just being the most extreme stars in a single, mainly prograde, disk population.

Thus far, we have neglected the effects of the $v_{z}$-velocity component. It does not, in fact, make that much difference: if we broaden the model spectra $S_{\alpha \beta \gamma}$ with an extra Gaussian dispersion $\sigma_{z 0} e^{-R / 2 h} \cos i$, which represents an exponentially declining vertical velocity dispersion as seen in nearby face-on galaxies (e.g., van der Kruit \& Freeman 1986), and attempt to reconstruct our absorption-line spectra from these components, essentially the same $f\left(E_{p}, L\right)$ is recovered. For example, the dashed contours in Figure 10 show the planar DF obtained for a vertical broadening with $\sigma_{z 0}=100 \mathrm{~km} \mathrm{~s}^{-1}$, and
Figure 11 shows the corresponding $v_{\phi^{-}}, v_{R^{-}}$, and $v_{z^{-}}$ distributions. The counterrotating fraction of stars does begin to decline significantly for $\sigma_{z 0} \gtrsim 100 \mathrm{~km} \mathrm{~s}^{-1}$. However, the goodness of fit is substantially worse for such large vertical dispersions since the high-velocity wing of the LOSVDs is too steep to be consistent with such a large vertical broadening: $\chi^{2}$ for such a model exceeds the value for the fit with no vertical broadening by $\gtrsim 84$; if the fits were both good, then this difference would be distributed as $\chi^{2}(v=1)$. From the models which fit the data adequately, we find that the possible range for the fraction of counterrotating stars is between $20 \%$ and $30 \%$. In all the successful models, this fraction is approximately independent of radius.

\subsection{Bilinear Segments}

Although the ellipsoidal basis functions provide a smooth description of the DF and can be treated analytically, they suffer from the drawback that they are far from orthogonal. 
Thus different components can be highly correlated, which renders the minimization problem somewhat ill-conditioned and ultimately leads to the breakdown of the quadratic programming algorithm. Another problem is the need for a dense grid on which to test the positivity constraints, which adds greatly to the computational expense. We now describe an alternative set of basis functions which, though less analytically tractable, are almost as orthogonal as is physically possible, and which require only a minimal set of positivity constraints.

Each element of this basis set is a "fat" orbit. In other words, each component is a particular orbit together with smaller contributions from its neighbors in phase space. Since the properties of the orbits are the fundamental characteristic of the problem, it is not possible to construct components which are less correlated than the orbits themselves without having some elements with (unphysical) negative occupation numbers.

A simple set of fat orbits can be constructed as follows. We divide the integral plane $\left(\left\{2\left[E_{p}-E_{\text {circ }}(L)\right]\right\}^{1 / 2}, L\right)$ into a hexagonal grid. Each component is then based on a single grid point, where we set the phase-space density to unity; at all other grid points it is zero, and between grid points the DF is given by linear interpolation on the triangular grid cells. A few such components are illustrated in Figure 12. Thus each component is quite compact in phase space, with its extent governed by the chosen hexagonal grid. A linear combination of these fat orbits is a triangular tesselation of the integral plane, with the coefficients of each component giving the phase-space density at the corresponding grid point and the DF linearly interpolated on triangular segments joining neighboring grid points. The positivity constraint simply becomes the requirement that each coefficient is nonnegative-there are thus only as many constraints as components. The smoothness scale in the integral plane is set explicitly by the grid in both coordinates (in the case of the ellipsoidal components, smoothness was only imposed in the $L$-direction).

The DF obtained from the spectra of NGC 7217 using a grid of 45 such components is illustrated in Figures 13 and 14.

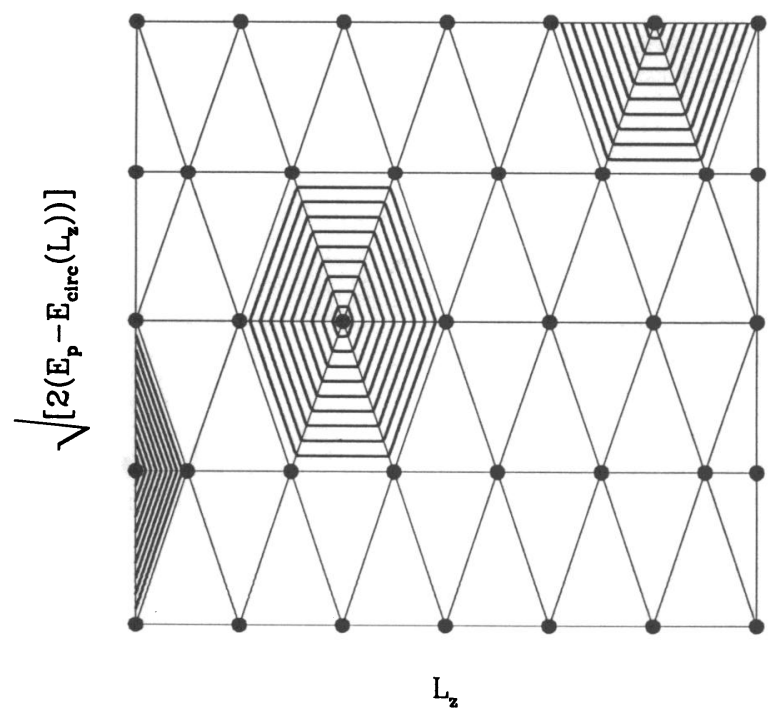

FIG. 12.-Some of the "fat orbit" basis functions used in the reconstruction of the DF using bilinear segments. The grid is outlined with thin lines, and the fat orbit DFs centered on three grid points are contoured. Sums of such orbits describe a general bilinear tesselation of this grid.

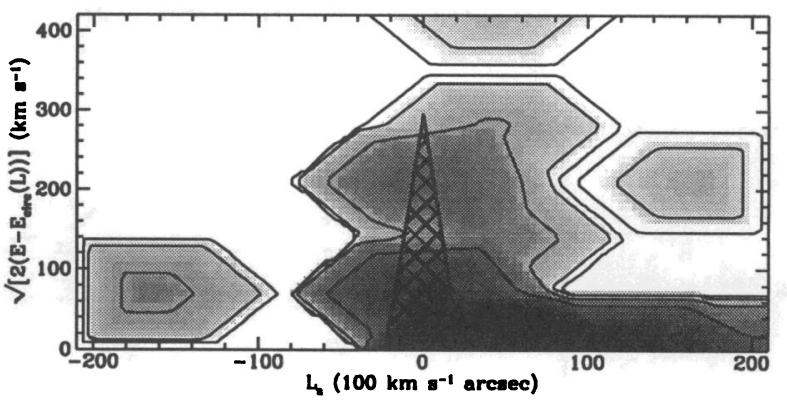

Fig. 13.-Best-fit bilinear-segment DF fitted to the spectra of NGC 7217 with $R>10^{\prime \prime}$ assuming zero vertical velocity dispersion (cf. Fig. 10).

Comparing Figures 10 and 13, it is clear that the fat orbits do not smooth the DF as much as the ellipsoidal components. As might be expected for these higher resolution components, the derived DF is somewhat noisier, but the main features are reassuringly comparable; if we were to smooth Figure 13 to the same resolution as Figure 10, then the DFs would appear very similar. The highest density of stars in both DFs are on prograde low-eccentricity orbits (bottom right of Figs. 10 and 13), with a tail of populated orbits at higher eccentricity, particularly those with lower angular momenta. This analysis also confirms the reality of the counterrotating population: like the UGD analysis, the dynamical model-fitting returns an estimate of the error on the DF at each point, and the component at the lower left of Figure 13 is nonzero at a formal significance level of $6 \sigma$. The low eccentricity of this component also implies that it represents a regular disk population with a relatively small radial dispersion.

\section{DISCUSSION}

Analysis of high signal-to-noise spectra of the normal Sab galaxy NGC 7217 has turned up the surprising result that a sizeable fraction $(\sim 30 \%)$ of the stars in the disk of this galaxy are on retrograde orbits. Fitting detailed dynamical models directly to the spectra confirms that these stars exist and that they seem to constitute a kinematically distinct component. This galaxy is not unique, as two distinct stellar components rotating in opposite directions have recently been discovered in NGC 4550, an S0 system (Rubin et al. 1992; Rix et al. 1992). In the case of NGC 4550, the prograde and retrograde components contain almost equal numbers of stars, and so the counterrotation was evident directly from the stellar spectra; what is particularly interesting about NGC 7217 is that one component is significantly stronger than the other, and so the retrograde component was only revealed by a detailed analysis of the LOSVD.

It is quite possible that many galaxies contain similar dynamical oddities which would not have been uncovered by the analysis of lower signal-to-noise data or by a traditional single-Gaussian fit to the LOSVD parameters. This possibility is made all the more credible since there is very little in the gross properties of NGC 7217 (see Table 1) that distinguishes it from other disk galaxies. The disk scale length and central surface brightness fall close to the center of the distribution for disk systems (van der Kruit 1986). The bulge-to-disk ratio is a little higher than the average for Sab or Sb galaxies, but not unusually so, and the total brightness falls within $1 \sigma$ of the mean for these types (Simien \& de Vaucouleurs 1986). Based on Matthewson, Ford, \& Buchhorn's (1992) calibration of the Tully-Fisher (1977) relation, the rotation speed and redshift of 

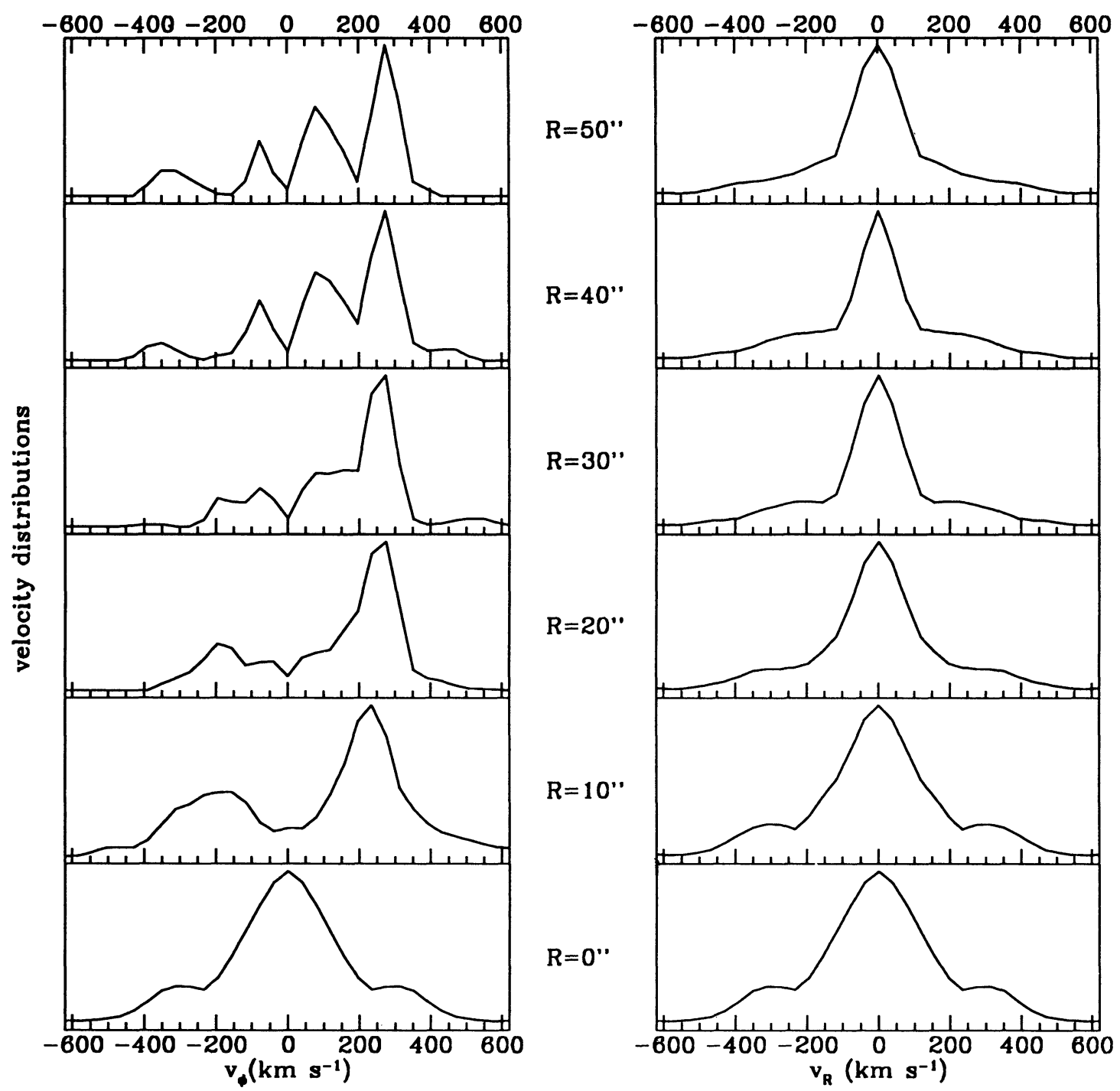
fits.

Fig. 14.-Individual components of the velocity distribution corresponding to the DF shown in Fig. 13 (cf. Fig. 11). No vertical motions were assumed for these

NGC 7217 predict an I-band magnitude for the system of $8.4 \pm 0.25$. This value conflicts somewhat with Kent's (1986) $r$-band magnitude measurement of 9.9 since typical $r-I$ colors for disk galaxies are only 0.5 . However, NGC 7217 is sufficiently close that its redshift is not dominated by the Hubble flow, and so there are substantial uncertainties in its distance. The combination of this factor, uncertainties in the galaxy's $r-I$ color, and the intrinsic scatter in the TullyFisher relation mean that the discrepancy is not very significant.

Beside the low-surface-brightness ring in this galaxy noted in the introduction, which may provide some clue to the origin of the counterrotating component, the only other unusual feature of NGC 7217 is that it has a very low atomic hydrogen (H I) content. This peculiarity is illustrated in Figure 15, which shows that NGC 7217's total H I mass lies almost an order of magnitude below the average for disk galaxies of similar types. Of course, our selection of NGC 7217 on the basis of its regular appearance has probably biased us toward galaxies containing less cool gas, and this bias complicates assessment of the sig- nificance of the low gas fraction. Nevertheless, there are good reasons why we might expect a galaxy containing counterrotating populations to have a low $\mathrm{H}$ i content. In particular, stellar material lost from the two components due to winds and novae will collide head-on, injecting energy into the inter-

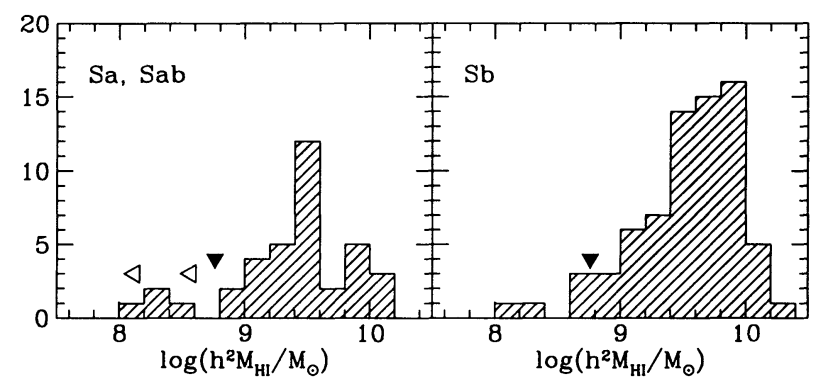

Fig. 15.-Distribution of total $\mathrm{H}$ I mass for a sample of $\mathrm{Sa}-\mathrm{Sb}$ galaxies, compiled by Haynes \& Giovanelli (1984). The value for NGC 7217 is indicated by the filled triangle. Upper limits due to nondetections for two galaxies are indicated as open triangles. 
stellar medium. This heating process will partially ionize the interstellar medium, which goes some way toward explaining the low un-ionized hydrogen mass. The resulting hot component of the interstellar medium will produce an enhanced soft X-ray flux akin to what is seen in elliptical galaxies (Kim, Fabbiano, \& Trinchieri 1992), and so X-ray luminosity might prove to be a useful diagnostic when searching for disk galaxies with counterrotating disks.

One other aspect of counterrotation in disk galaxies which requires some analysis is the dynamical stability of such systems. Interestingly, the counterrotating streams can act to remove the gravitational instabilities that generate spiral structure: essentially, the instabilities that start to grow in the two different populations destructively interfere with each other, suppressing the growth of unstable modes and resulting at best in a mottled interference pattern (Kalnajs 1977). This suppression is intuitively apparent in the extreme case of a galaxy with equal populations of stars rotating in each direction, as such a system would not have a preferred direction in which its spiral arms can trail. In this context, it is interesting to note that NGC 7217's sense of rotation is not immediately obvious from its flocculent spiral structure. As well as suppressing instabilities, a counterrotating population can also introduce some new ones such as the two-stream vertical instability described by Toomre (1966). Detailed investigations of such instabilities in the context of counterrotating disk galaxies are currently in progress (Merritt \& Sellwood, private communication).

The obvious remaining question that the peculiar dynamics of NGC 7217 raises is how such a counterrotating system could form. One possibility is that it results from a merger between two disk galaxies with their spins initially antiparallel. However, simulations show that such mergers greatly heat the stellar population and hence produce an excessively thick disk or disrupt it entirely (see, e.g., Barnes 1992; Tóth \& Ostriker 1992). A more successful model comes from noting that intergalactic material continues to rain down on a galaxy throughout its lifetime, and that cosmological models predict that the angular momentum of this matter relative to the spin of the galaxy will vary with time (Ryden 1988). Furthermore, the characteristic timescale for reversal in the direction of the accreting material's angular momentum is significantly less than the age of the universe (Quinn \& Binney 1992). A scenario to explain a counterrotating stellar disk then suggests itself: an initial gas disk forms from infalling material and mostly becomes converted into stars as in a normal spiral galaxy. If the direction of the angular momentum of further infalling gas varies slowly with time, then the disk will adjust its own orientation to absorb the new material. However, a rapid, substantial change in accreting angular momentum will result in a new gas disk forming in a different plane to the main galaxy. Any remaining gas in the original disk will collide with the new material, and the resulting cancellation of angular momentum will then dump matter into the center of the galaxy where presumably it will be converted into stars, resulting in the large observed stellar bulge. This dumping process may also be partly responsible for the relative $\mathrm{H}_{\mathrm{I}}$ deficiency in the disk of NGC 7217. After the residual first-generation gas has been swept aside, the remaining gas in the new disk can settle down to making stars. ${ }^{2}$ If the two disks are almost orthogonal then a polar-ring galaxy will result - an arrangement which can be long-lived (Christodoulou et al. 1992)-but otherwise collisionless dissipation between the two interpenetrating disks will cause them to settle down to a single plane with antiparallel spins. It is possible that the outer ring of NGC 7217 is also a product of this evolutionary sequence; unfortunately its surface density was too low for us to be able to measure its kinematics. Further support for this scenario comes from the existence of polar-ring galaxies (for a review see Casertano et al. 1991), and from the discovery of counterrotating gas disks in the centers of many S0 galaxies (Galletta 1987; Bettoni et al. 1990, 1991; Bertola et al. 1992). As discussed extensively by Bettoni \& Galletta (1991), it is difficult to see how these disks could be formed in any way other than through secondary infall of misaligned angular momentum material.

Note that our requirement that the accreted material be gaseous (collisionless matter will not dissipate and settle down into a disk) also provides the mechanism whereby the usual argument against substantial secondary infall onto galactic disks (Tóth \& Ostriker 1992) can be circumvented. Infalling gas actually thins the disk, since as the accreted gas settles in the galactic plane it adiabatically pulls in the preexisting stellar component.

Finally, we note that two of the observational properties that are strongly affected by this evolutionary scenario - the size of the stellar bulge, which is enhanced by the dumping of material in the center of the galaxy, and the strength of spiral structure, which is suppressed by the counterrotating stellar populations-are exactly the properties that Hubble (1936) used for his classification of disk galaxies. We have also argued that similar counterrotating populations in many other disk galaxies would probably not have been detected in existing kinematic observations. It is therefore quite possible that the Hubble sequence for disk galaxies, $\mathrm{S} 0 \rightarrow \mathrm{Sd}$, might be partly attributable to the magnitude of currently undetected counterrotating stellar populations in these systems. This hypothesis can be tested directly by obtaining high signal-to-noise spectra of more disk galaxies with a range of morphologies and seeing whether their velocity distributions imply counterrotating components whose size correlates with the Hubble type of the galaxy. We are presently engaged in such a survey.

This paper is based on data obtained using the CanadaFrance-Hawaii Telescope, which is operated by CNRS of France, NRC of Canada, and the University of Hawaii. Data analysis was performed using IRAF, which is distributed by National Optical Astronomy Observatories in cooperative agreement with the National Science Foundation. The research was supported by SERC grant GR/H 84253 (M. R. M.), and by a Hubble Fellowship through grant HF1020.01-91A awarded by the Space Telescope Science Institute (which is operated by the Association of Universities for Research in Astronomy, Inc., for NASA under contract NAS 5-26555) (K. K.).

\footnotetext{
${ }^{2}$ In the case of NGC 7217, where the residual gas rotates in the same direction as the dominant disk component, we can conclude that the smaller component must have formed first.
} 
Barnes, J. E. 1992, ApJ, 393, 484

Bender, R. 1988, A\&A, 202, L5

1990, A\&A, 229, 441

Bertola, F, \& Bettoni, D 1988, ApJ, 329,102

Bertola, F., Buson, M., \& Zeiliger, W. W. 1992, ApJ, 401, L79

Bettoni, D., Fasano, G., \& Galletta, G. 1990, AJ, 99, 1789

Bettoni, D., \& Galletta, G. 1991, in Dynamics of Disc Galaxies, ed.

B. Sundelius (Göteborg: Göteborg Univ. and Chalmers Univ. Technology), 317

Bettoni, D., Galletta, G., \& Oosterloo, T. 1991, MNRAS, 248, 544

Binney, J., \& Tremaine, S. 1987, Galactic Dynamics (Princeton: Princeton Univ. Press), 22

Casertano, S., Sackett, P., \& Briggs, F., eds. 1991, Warped Disks and Inclined Rings Around Galaxies (Cambridge: Cambridge Univ. Press)

Christodoulou, D. M., Katz, N., Rix, H.-W., \& Habe, A. 1992, ApJ, 395, 113

Dejonghe, H. 1989a, ApJ, 343, 113

Dejonghe, H. 1989b, in Dynamics of Dense Stellar Systems, ed. D. Merritt (Cambridge: Cambridge Univ. Press)

de Vaucouleurs, G., de Vaucouleurs, A., \& Corwin, H. G., Jr. 1976, Second Reference Catalogue of Bright Galaxies (Austin: Univ. Texas Press)

Erickson, R. R. 1975, ApJ, 195, 343

Franx, M., \& Illingworth, G. D. 1988, ApJ, 327, L55

Galletta, G. 1987, ApJ, 318, 531

Haynes, M. P., \& Giovanelli, R. 1984, AJ, 89, 758

Hubble, E. 1936, The Realm of the Nebulae (New Haven: Yale Univ. Press)

Jedrzejewski, R., \& Schechter, P. L. 1989, AJ, 98, 147

Kalnajs, A. J. 1977, ApJ, 212, 637

Kent, S. M. 1986, AJ, 91, 1301

Kim, D.-W., Fabbiano, G., \& Trinchieri, G. 1992, ApJ, 393, 134
REFERENCES

Kormendy, J. 1984, ApJ, 287, 577

Kuijken, K. 1991, ApJ, 372, 125

Kuijken, K., \& Merrifield, M. R. 1993, MNRAS, 264, 712 (Paper I)

Lacey, C. G. 1991, in Dynamics of Disk Galaxies, ed. B. Sundelius (Göteborg: Göteborg Univ. and Chalmers Univ. Technology), 257

Matthewson, D. S., Ford, V. L., \& Buchhorn, M. 1992, ApJS, 81, 413

Merrifield, M. R. 1993, MNRAS, 261, 233

Merritt, D., \& Saha, P. 1993, ApJ 409,75

Peterson. C. J. Rubin V C. Ford W. K \& Roberts, M. S. 1978, ApJ, 226, 770

Quinn, T., \& Binney, J. 1992, MNRAS, 255, 729

Rix, H.-W., Franx, M., Fisher, D., \& Illingworth, G. 1992, ApJ, 400, L5

Rix, H.-W., \& White, S. D. M. 1992, MNRAS, 254, 389

Rubin, V., Graham, J., \& Kenney, J. 1992, ApJ, 394, L9

Ryden, B. 1988, ApJ, 329, 589

Saha, P. \& Williams, T. B. 1993, CITA preprint

Sandage, A., \& Tammann, G. A. 1987, A Revised Shapley-Ames Catalog of Bright Galaxies, (Washington DC: Carnegie Inst. Washington)

Sargent, W. L. W., Schechter, P. L., Boksenberg, A., \& Shortridge, K. 1977, ApJ, 212, 326

Simien, F., \& de Vaucouleurs, G. 1986, ApJ, 302, 564

Tonry, J., \& Davis, M. 1979, AJ, 84, 1511

Toomre, A. 1966, in Notes on the 1966 Summer Study Program in Geophysical Fluid Dynamics, (Woods Hole, MA: Woods Hole Oceanographic Inst.), 111

Tóth, G., \& Ostriker, J. P. 1992, ApJ, 389, 5

Tully, R. B., \& Fisher, J. R. 1977, A\&A, 54, 661

van der Kruit, P. C. 1986, A\&A, 173, 59

van der Kruit, P. C., \& Freeman, K. C. 1986, ApJ, 303, 556

van der Marel, R. P., \& Franx, M. 1993, ApJ, 407, 525 